\title{
Crosstalk Reduction in Epimysial EMG Recordings from Transhumeral Amputees with Principal Component Analysis
}

\author{
A. Matran-Fernandez ${ }^{1}$, E. Mastinu ${ }^{2}$, R. Poli ${ }^{1}$, M. Ortiz-Catalan ${ }^{3}$, L. Citi $^{1}$
}

\begin{abstract}
Electromyographic (EMG) recordings of muscle activity using monopolar electrodes suffer from poor spatial resolution due to the crosstalk from neighbouring muscles. This effect has mainly been studied on surface EMG recordings. Here, we use Principal Component Analysis (PCA) to reduce the crosstalk in recordings from unipolar epimysial electrodes implanted in three transhumeral amputees. We show that the PCA-transformed signals have, on average, a better signal-tonoise ratio than the original unipolar recordings. Preliminary investigations show that this transformation is stable over long periods of time. If the latter is confirmed, our results show that the combination of PCA with unipolar electrodes allows for a higher number of muscles to be targeted in an implant (compared with bipolar electrodes), thus facilitating 1-to-1 proportional control of prosthetic hands.
\end{abstract}

\section{INTRODUCTION}

Electromyography (EMG) is the electrical recording of muscular activation. To record such activity electrodes may be placed on the skin (surface EMG; sEMG) or invasively sewn onto the muscle (e.g., epimysial EMG). EMG records activity from the muscle of interest together with contamination from other active neighbouring muscles (i.e., crosstalk), and other sources [1]. Studies of sEMG of different muscles and electrode configurations [2]-[4] suggested that the effects of crosstalk may significantly reduce the quality of the recordings. Crosstalk is much reduced when using bipolar electrodes. However, these have the disadvantage of needing two wires per recorded signal, as opposed to monopolar electrodes which only require one per site, plus a common reference. For this reason, techniques to reduce crosstalk in monopolar sEMG have been developed. The most popular one consists of subtracting the values of two or more nearby electrodes [4]-[6]. However, crosstalk reduction with this technique depends on factors such as the inter-electrode distance, with the larger the distance the less effective the technique [1], [2].

Invasive EMG also suffers from crosstalk. However, to the best of our knowledge, no work has been done to establish to what degree the signals are contaminated nor to reduce crosstalk. Yet, in the case of implanted electrodes, the use of

This work was supported by the European Commission under the DeTOP project (H2020-ICT-687905).

${ }^{1}$ A. Matran-Fernandez, L. Citi and R. Poli are with the School of Computer Science and Electronic Engineering, University of Essex, Wivenhoe Park, CO4 3SQ, Colchester, United Kingdom. Email: \{amatra, lciti, rpoli\}eessex.ac.uk

${ }^{2}$ E. Mastinu is with the Dept. of Electrical Engineering, Chalmers University of Technology, Gothenburg, Sweden. Email: en zo@ chalmers.se

${ }^{3} \mathrm{M}$. Ortiz-Catalan is with the Dept. of Electrical Engineering, Chalmers University of Technology; and Integrum AB, Gothenburg, Sweden. Email: maxodchalmers.se monopolar electrodes is even more compelling as there may be a limit in the number of wires that can go through the human-machine interface [7], which in turn places an upper bound on the amount of signals available. For this reason, it would be much preferable to implant monopolar electrodes if the quality of the EMG recorded was comparable to that of bipolar electrodes.

In this work, we record EMG from invasive unipolar electrodes in the muscles of the upper arm by transhumeral amputees. We used Principal Component Analysis (PCA) to improve the Signal-to-Noise Ratio (SNR) of unipolar electrodes and reduce the crosstalk present in the recordings. Lastly, we introduce a metric based on the coherency between pairs of electrodes to measure the crosstalk.

PCA [8], [9] is an unsupervised technique transforming a set of correlated variables into new orthogonal variables (principal components; PCs) that maximally capture the variance of the original data. Since PCs are sorted in decreasing order of variance, PCA can be used to reduce the dimensionality of a dataset by keeping only the first few PCs [9]. This is the main use of PCA with sEMG data, where the PCA-transformed EMG is used in pattern recognition approaches [9], [10], e.g., to control a robotic hand [11] and in studies of muscle strength, fatigue and muscle synergies (e.g., [10]; for a detailed survey refer to [9]). Similarly, blind source separation methods, such as independent component analysis, have been extensively used with high-density sEMG in order to reduce noise and decompose the sEMG into motor unit action potentials [12], [13]. However, no application of such techniques to improve EMG from implanted electrodes has been proposed.

\section{METHODS}

\section{A. Data}

The data used in this study had been previously collected in 2017 from three transhumeral amputees (P1, P2 and P3) that received the e-OPRA Implant System in 2014-2015 [7]. The study was approved by the Swedish Regional Ethical Committee in Gothenburg.

Each participant had four pairs of bipolar electrodes from which we recorded simultaneously using unipolar and bipolar configurations. P1 and P2 had two bipolar electrodes placed on the biceps and two more on the triceps. These patients underwent two recording sessions each, the main difference being the location of the reference electrode for the unipolar recordings. Only one recording session was available from P3, comprising two bipolar electrodes (one in the biceps and one in the triceps) and four unipolar electrodes. 
Participants were seated in a relaxed position in front of a computer and asked to follow the instructions presented on a screen in front of them. During each recording session, patients performed three repetitions of 3 seconds of contraction followed by 3 seconds of rest for four different movements: open/close hand and flex/extend elbow. Thus, for each movement each session contained 18 seconds of recording. The first 12 seconds of data from each movement were used to compute the PCA projections of each participant.

Data were collected at a sampling rate of $1 \mathrm{kHz}$ and bandpass filtered in the range $120-400 \mathrm{~Hz}$ using a $5^{\text {th }}$ order zerophase band-pass Butterworth filter.

\section{B. Methods for Assessing the Degree of Crosstalk}

Traditionally, crosstalk has been measured by means of the value of the peak of the cross-correlation between a pair of electrodes, typically placed on a muscle and its antagonist [2], [14]. We denote this as $P_{x}$ and report this amount normalised by the standard deviation of the signals.

However, $P_{x}$ alone does not take into account whether the similarity between the two signals is due to crosstalk or to muscle co-activation [14]. For this reason, here we propose to use also a metric based on the coherency between each pair of electrodes. This is described below.

Given two signals, $x(t)$ and $y(t)$, their coherency is $R(f)=C_{x y}(f) / \sqrt{C_{x x}(f) \cdot C_{y y}(f)}$, where $C_{x y}(f)$ represents the (complex) cross-spectrum between the two signals, and $C_{x x}(f)$ and $C_{y y}(f)$ are the spectra for $x(t)$ and $y(t)$, respectively [15, ch. 7]. Assuming that volume conduction is dominated by the resistive component, the real part of $R(f)$ is predominantly due to volume conduction (i.e., crosstalk), whereas the imaginary part is mostly sensitive to interaction between two processes that are time-lagged to each other (e.g., co-activation and a common neural drive) [16].

We calculated $R(f)$ for each pair of electrodes of a recording session using a 3 -second sliding Hanning window across the test set with an overlap of $25 \%$ (i.e., the window was shifted by $2.25 \mathrm{~s}$ ). This gives a series of coherency values (for a range of frequencies and over the length of the recording) as a result. We summarise them into a single crosstalk measure using the 75-th percentile of the distribution of the real part of the coherency for each pair of electrodes, $C_{75}$. We also report the Real-to-Imaginary Ratio (RIR), which is the fraction of points for which the absolute value of the real part of $R(f)$ is larger than the absolute value of the imaginary part (w.r.t. the whole set of points obtained for that electrode pair).

Lastly, we use the SNR to measure the degree to which the PCA-transformed unipolar signals approximate the simultaneously recorded bipolar recordings. SNR is the ratio between the power of the signal $s(t)$ and the power of the noise $n(t)$, which are given by

$$
s(t)=\frac{\operatorname{Cov}[p(t), b(t)]}{\operatorname{Var}[b(t)]} \cdot b(t) \quad \text { and } \quad n(t)=p(t)-s(t)
$$

where $p(t)$ is a PCA-transformed unipolar recording and $b(t)$ the corresponding bipolar recording. The improvement

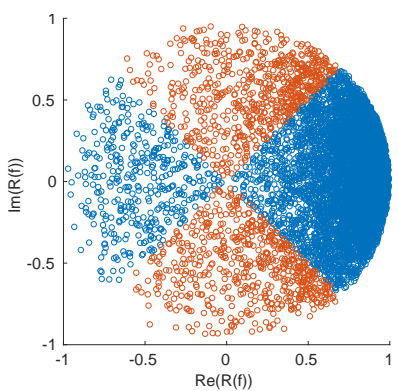

(a)

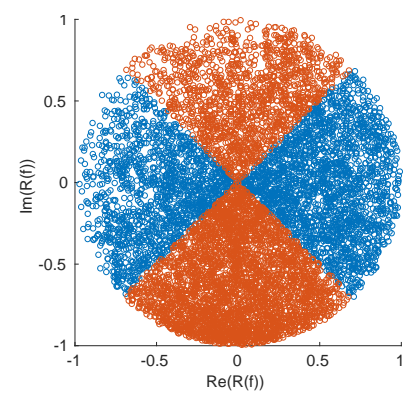

(b)
Fig. 1. Coherency represented as a 2D diagram for (a) a pair of electrodes with crosstalk and (b) a pair of electrodes with no crosstalk.

in the quality of the signals after PCA was measured as $\mathrm{SNR} / \mathrm{SNR}_{0}$, i.e., the ratio between the SNR of a transformed electrode relative to that of the standard (unipolar) recording obtained from that electrode, $\mathrm{SNR}_{0}$. Values of $\mathrm{SNR} / \mathrm{SNR}_{0}>1$ indicate a higher SNR after PCA.

\section{RESULTS}

\section{A. Crosstalk in Epimysial Recordings}

We start by justifying the use of RIR and $C_{75}$, derived from the coherency, as metrics for measuring the crosstalk and then we analyse the amount of crosstalk present in our recordings using these metrics and $P_{x}$.

Coherency can be visualised on a $2 \mathrm{D}$ plane where the real and imaginary parts form a coordinate system. Fig. 1 shows two examples of coherency plots for two different pairs of electrodes. Most of the points in Fig. 1(a) are located on the rightmost part of the plot, corresponding to high values of the real part of coherency. Since the real part of the coherency is dominated by conduction effects (Section II-B), this plot corresponds to a pair of electrodes in which there is crosstalk. Conversely, the plot in Fig. 1(b) has a large fraction of points towards $\operatorname{Re}(R(f))=0$ and $\operatorname{Im}(R(f))=-1$, thus representing a case where there was co-activation of the muscles but no crosstalk. Visual inspection of the raw traces confirmed these observations.

The blue (resp. red) points in the plots from Fig. 1 represent the values of coherency for which the absolute value of the real part is larger (resp. smaller) than the absolute value of the imaginary part. In general, while co-activation leads to coherency spread across all phases, the coherency plot for a pair of electrodes with crosstalk due to resistive volume conduction will have the majority of the points with a dominant real component (blue-shaded areas in the figure). Hence, calculating the ratio of points that fall in the blue area with respect to the total number of points is a valid metric to measure the crosstalk and it is, indeed, what RIR represents. Moreover, since RIR takes into account the real and imaginary parts of the coherency, it allows to distinguish between co-activation (low values of RIR) and crosstalk (high values of RIR).

The $C_{75}$ measure defined in Section II-B is a summary of the tendency of absolute real values of coherency to approach 1 in the presence of crosstalk. Since $R(f)$ is calculated across the whole test set, but crosstalk may only be present in a 
TABLE I

Average RIR, $C_{75}$ AND $P_{x}$ FOR EACH ReCORding SESSION ACROSS ALL PAIRS OF UNIPOLAR AND BIPOLAR ELECTRODES.

\begin{tabular}{|c|c|c|c|c|c|c|}
\hline ID & $\mathrm{RIR}_{\mathrm{u}}$ & $\mathrm{RIR}_{\mathrm{b}}$ & $C_{75, \mathrm{u}}$ & $C_{75, \mathrm{~b}}$ & $P_{x, \mathrm{u}}$ & $P_{x, \mathrm{~b}}$ \\
\hline P1-1 & 0.53 & 0.50 & 0.61 & 0.58 & 0.055 & 0.045 \\
P1-2 & 0.54 & 0.51 & 0.62 & 0.57 & 0.047 & 0.034 \\
P2-1 & 0.68 & 0.52 & 0.79 & 0.58 & 0.450 & 0.180 \\
P2-2 & 0.63 & 0.54 & 0.71 & 0.63 & 0.289 & 0.205 \\
P3-1 & 0.51 & 0.49 & 0.55 & 0.55 & 0.056 & 0.018 \\
\hline \hline Mean & 0.58 & 0.52 & 0.66 & 0.58 & 0.179 & 0.096 \\
\hline
\end{tabular}

subset of movements, we chose the 75-th percentile (i.e., the third quartile) as it gives an indication of the presence of crosstalk somewhere in the recording while still being robust to spurious values.

The values of the $C_{75}$ and RIR for the two examples in Fig. 1 are (a) $C_{75}=0.86, \mathrm{RIR}=0.81$ and (b) $C_{75}=0.58$, $\mathrm{RIR}=0.41$. Table I reports the average RIR, $C_{75}$ and $P_{x}$ separately for unipolar and bipolar electrodes and for each recording session. The first thing to notice from this table is the fact that recording sessions P1-1, P1-2 and P31 have almost no crosstalk, indicated by the similar RIR values obtained for the unipolar $\left(\mathrm{RIR}_{\mathrm{u}}\right)$ and corresponding bipolar $\left(\mathrm{RIR}_{\mathrm{b}}\right)$ pairs of electrodes. Moreover, as expected, the values of all measures considered are higher for the unipolar recordings than for the bipolar recordings, showing that there is less crosstalk in the latter.

Lastly, it should be noted that the columns for unipolar and bipolar electrodes of Table I represent upper and lower bounds, respectively, for each of the metrics. The values for the bipolar recordings are the ones we aim for using PCA, whereas the ones obtained for the unipolar electrodes represent the baseline condition.

\section{B. Crosstalk Reduction via PCA}

Fig. 2 shows the PCs needed to transform the unipolar recordings for the two different recording sessions of participant P2. For ease of interpretation, the channels (i.e., the $\mathrm{x}$-axis) have been reorganised so that the main diagonal of each matrix contains the largest (in absolute value) values of each column. The biggest (in absolute value) coefficients off-diagonal coefficients in Fig. 2(a) are on the top left corner. Moreover, those coefficients have approximately the same value with opposed sign. This can be interpreted as a projection of channels 1 and 2 onto a new coordinate system that is tilted $45^{\circ}$ with respect to the original one (i.e., a spatial differentiation). In a way, this is no different from traditional methods to undo the crosstalk in sEMG recordings [4] in which a sort of bipolar derivation (component 2 in this figure) is done to enhance the EMG signal. The difference in this case is that the distance between electrodes 1 and 2 is not in the order of millimetres as in sEMG arrays or bipolar configurations. Rather, they are located in the triceps and biceps, respectively. The third component, instead, approximately performs the average of channels 1 , 2 and 3, and subtracts it from channel 4, which is loosely equivalent to performing a common average reference (i.e.,

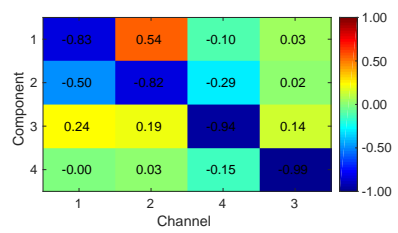

(a)

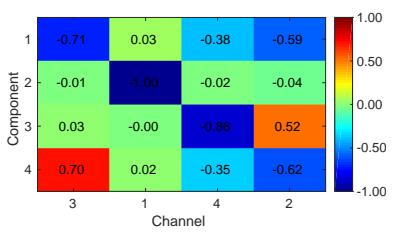

(b)
Fig. 2. PCA coefficients obtained for the two recording sessions from participant P2: (a) P2-1 and (b) P2-2. Where needed, the channels have been reorganised so that the main diagonal of each matrix contains the largest (in absolute value) values of each column.

TABLE II

Average RIR, $C_{75}, P_{x}$ And Ratio SNR/SNR 0 FOR Each RECORDING SESSION AFTER PCA.

\begin{tabular}{|c|c|c|c|c|}
\hline ID & RIR & $C_{75}$ & $P_{x}$ & SNR/SNR $_{0}$ \\
\hline P1-1 & 0.55 & 0.63 & 0.053 & 0.92 \\
P1-2 & 0.55 & 0.62 & 0.047 & 0.98 \\
P2-1 & 0.61 & 0.71 & 0.405 & 1.59 \\
P2-2 & 0.61 & 0.70 & 0.113 & 1.67 \\
P3-1 & 0.50 & 0.54 & 0.046 & 2.04 \\
\hline \hline Mean & 0.56 & 0.64 & 0.133 & 1.44 \\
\hline
\end{tabular}

it finds the background noise and removes it). Conversely, the PCs shown in Fig. 2(b) are more complex. The third PC implements the same sort of spatial filter that we observed above, in this case between the two electrodes placed on the biceps. However, the first and last components involve a more sophisticated derivation obtained from channels $2-4$. In particular, the last PC represents the same structure as a double-differential filter [4] between the triceps (electrode 3) and the two electrodes from the biceps.

Table II summarises the average RIR, $C_{75}$ and peak of the cross-correlation for each recording session, after PCA transformation. As expected, the average values at the bottom of the table are between the respective upper and lower bounds observed in Table I.

A row-by-row analysis shows that the biggest reduction in crosstalk is obtained for the data from participant P2, which makes sense given that these were the ones where the crosstalk was most prominent according to the baseline measurements. Even though the mean values reported do not seem much different from the baseline, the improvements can be seen very clearly in the last column of Table II, which shows increases in the SNR of up to twice the original value. It should be noted, however, that the averages reported above and in Table II include cases where no crosstalk was present, so there were no changes before and after the transformation. To really measure the extent of the improvements brought upon by PCA, we established a threshold at RIR $=0.6$ and considered the number of times that RIR decreased after PCA in those cases in which RIR was above this pre-defined threshold. Out of the 12 pairs of electrodes (across all datasets) for which RIR was above this threshold $\left(\mu_{\mathrm{RIR}, \text { before }}=0.68\right)$, it decreased after PCA in 10 of them $\left(\mu_{\mathrm{RIR}, \text { after }}=0.58\right)$. Similarly, the $C_{75}$ from those 12 pairs of electrodes decreased from an average of 0.79 to 0.68 after PCA. For instance, for the pair of electrodes depicted 

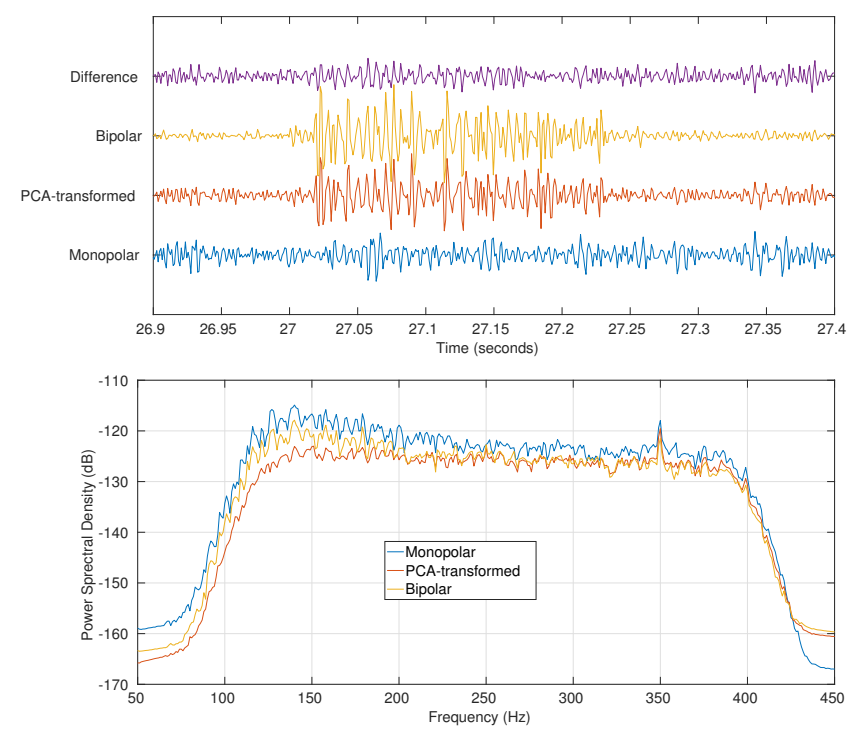

Fig. 3. Top: epimysial EMG signal before and after PCA transformation, together with the corresponding bipolar recording and the difference between the PCA-transformed unipolar and the bipolar. Bottom: Power spectral density for the raw (after preprocessing) and transformed unipolar recording and the bipolar EMG.

in Fig. 1(a) (i.e. the pair of electrodes with the highest degree of crosstalk amongst all the recordings) the baseline $\mathrm{RIR}=0.81$ decreased to $\mathrm{RIR}=0.61$ after PCA.

Fig. 3 shows a fragment of EMG signal recorded from one electrode before and after PCA transformation, as well as the corresponding bipolar recording and the difference between them. The variance of the difference signal does not change between the resting periods (beginning and end of the fragment shown) and the period of muscle activation shown in the middle of the recording. Together with the power spectral densities (Fig. 3(bottom)) also shown in the figure for the unipolar electrode before and after PCA and the bipolar one, it can be seen that the PCA transformation successfully reduces the crosstalk (as evidenced by the decrease in RIR, $C_{75}$ and $P_{x}$ values) without significantly distorting the underlying EMG. Finally, we measured the value of the 75-th percentile of the imaginary part of the coherency for each pair of electrodes before and after transformation in order to assess whether $\operatorname{Im}(R(f))$ (and, thus, the part that reflects co-activation of muscles rather than crosstalk) was affected by the PCA transformation. A paired Wilcoxon signed-rank test for the difference in medians confirmed that the distributions were not statistically different $(p=0.41)$.

\section{DISCUSSION AND CONCLUSIONS}

In this work, we used PCA to reduce crosstalk in unipolar epimysial recordings on transhumeral amputees. We introduced RIR as a metric to discriminate between the real and imaginary parts of the coherency, which represent crosstalk and co-activation, respectively. We showed that it can be used to discriminate between recordings with and without crosstalk.
Using RIR and other traditional metrics to measure crosstalk, we also showed that PCA effectively reduces the crosstalk in pairs of electrodes, and that it does not affect the imaginary part of the coherency. That is, coactivation registered in the EMG was not destroyed by the transformation. We plan to further explore these results on a larger pool of amputees.

Moreover, preliminary analyses with data recorded over a period of years show that PCA transformations are stable over time, managing to reduce the crosstalk across sessions taken months apart, which is remarkable considering the limited amount of data used to train the model. We plan to investigate this, together with other methods to reduce crosstalk (e.g., common spatial patterns), in future research.

\section{REFERENCES}

[1] C. J. De Luca, M. Kuznetsov, L. D. Gilmore, and S. H. Roy, "Interelectrode spacing of surface EMG sensors: Reduction of crosstalk contamination during voluntary contractions," Journal of Biomechanics, vol. 45 , no. 3, pp. 555-561, 2012.

[2] D. Winter, A. Fuglevand, and S. Archer, "Crosstalk in surface electromyography: Theoretical and practical estimates," Journal of Electromyography and Kinesiology, vol. 4, no. 1, pp. 15-26, 1994.

[3] T. M. Vieira, A. Botter, S. Muceli, and D. Farina, "Specificity of surface EMG recordings for gastrocnemius during upright standing," Scientific reports, vol. 7, no. 1, p. 13300, 2017.

[4] J. Van Vugt and J. Van Dijk, "A convenient method to reduce crosstalk in surface EMG," Clinical Neurophysiology, vol. 112, no. 4, pp. 583$592,2001$.

[5] C. Disselhorst-Klug, J. Silny, and G. Rau, "Improvement of spatial resolution in surface-EMG: a theoretical and experimental comparison of different spatial filters," IEEE Transactions on Biomedical Engineering, vol. 44, no. 7, pp. 567-574, 1997.

[6] T. J. Koh and M. D. Grabiner, "Cross talk in surface electromyograms of human hamstring muscles," Journal of Orthopaedic Research, vol. 10, no. 5, pp. 701-709, 1992.

[7] M. Ortiz-Catalan, B. Håkansson, and R. Brånemark, “An osseointegrated human-machine gateway for long-term sensory feedback and motor control of artificial limbs," Science Translational Medicine, vol. 6, no. 257, pp. 257re6-257re6, 2014.

[8] K.-L. Du and M. Swamy, "Principal component analysis," in Neural Networks and Statistical Learning. Springer, 2014, pp. 355-405.

[9] G. R. Naik, S. E. Selvan, M. Gobbo, A. Acharyya, and H. T. Nguyen, "Principal component analysis applied to surface electromyography: a comprehensive review," IEEE Access, vol. 4, pp. 4025-4037, 2016.

[10] G. C. Matrone, C. Cipriani, E. L. Secco, G. Magenes, and M. C. Carrozza, "Principal components analysis based control of a multidof underactuated prosthetic hand," Journal of NeuroEngineering and Rehabilitation, vol. 7, no. 1, p. 16, Apr 2010.

[11] K. Xing, P. Yang, J. Huang, Y. Wang, and Q. Zhu, "A real-time EMG pattern recognition method for virtual myoelectric hand control," Neurocomputing, vol. 136, pp. 345-355, 2014.

[12] M. Chen and P. Zhou, "A novel framework based on FastICA for high density surface EMG decomposition," IEEE Transactions on Neural Systems and Rehabilitation Engineering, vol. 24, no. 1, pp. 117-127, 2016.

[13] A. Holobar and D. Zazula, "Surface EMG decomposition using a novel approach for blind source separation," in Informatica Medica Slovenica, vol. 8, 2003, pp. 2-14.

[14] M. M. Lowery, N. S. Stoykov, and T. A. Kuiken, "A simulation study to examine the use of cross-correlation as an estimate of surface EMG cross talk," Journal of Applied Physiology, vol. 94, no. 4, pp. 13241334, 2003.

[15] D. R. Brillinger, Time series: data analysis and theory. Siam, 2001, vol. 36.

[16] G. Nolte, O. Bai, L. Wheaton, Z. Mari, S. Vorbach, and M. Hallett, "Identifying true brain interaction from EEG data using the imaginary part of coherency," Clinical neurophysiology, vol. 115, no. 10, pp. 2292-2307, 2004. 